\title{
Between the Paley-Wiener theorem and the Bochner tube theorem
}

\author{
by Zofia Szmydt and Bogdan Ziemian (Warszawa)
}

\begin{abstract}
We present the classical Paley-Wiener-Schwartz theorem [1] on the Laplace transform of a compactly supported distribution in a new framework which arises naturally in the study of the Mellin transformation. In particular, sufficient conditions for a function to be the Mellin (Laplace) transform of a compactly supported distribution are given in the form resembling the Bochner tube theorem [2].
\end{abstract}

1. Notation. We employ the usual notation of set theory. $\mathbb{R}$ denotes the set of real numbers, $\mathbb{R}_{+}$the set of positive real numbers, and $\mathbb{R}_{+}^{n}=\left(\mathbb{R}_{+}\right)^{n}$. For $x \in\left(x_{1}, \ldots, x_{n}\right) \in \mathbb{R}^{n}$ we set $\langle x\rangle=1+\left|x_{1}\right|+\ldots+\left|x_{n}\right|$. We write $x<y$ $(x \leq y)$ for $x, y \in \mathbb{R}^{n}$ to denote $x_{j}<y_{j}\left(x_{j} \leq y_{j}\right.$ resp. $)$ for $j=1, \ldots, n$, and we set $I=(0, t]=\left\{x \in \mathbb{R}^{n}: 0<x \leq t\right\}$, where $t \in \mathbb{R}_{+}^{n}$. By 1 we denote $(1, \ldots, 1) . \mathbb{N}$ is the set of positive integers and $\mathbb{N}_{0}$ the set of non-negative integers. We write $|\alpha|=\alpha_{1}+\ldots+\alpha_{n}$ for $\alpha \in \mathbb{N}_{0}^{n}$. For $x \in \mathbb{R}^{n}$ and $\alpha \in \mathbb{N}_{0}^{n}$ we write $x^{\alpha}=x_{1}^{\alpha_{1}} \ldots x_{n}^{\alpha_{n}}$.

We employ the usual notation of distribution theory. $D^{\prime}(\Omega)$ denotes the space of distributions on an open set $\Omega \subset \mathbb{R}^{n}$, and $D_{A}^{\prime}(\Omega)$ the space of distributions on $\Omega$ with support in $A \subset \Omega$. The value of a distribution $u$ on a test function $\varphi$ is denoted by $u[\varphi]$.

\section{Auxiliary theorems}

TheOREM 1. Let $u \in D_{K}^{\prime}\left(\mathbb{R}^{n}\right)$ where $K$ is a connected compact set in $\mathbb{R}^{n}$ such that any two points $x, y \in K$ can be joined by a rectifiable curve in $K$ of length $\leq \widetilde{C}|x-y|, \widetilde{C}<\infty$. Then there exists a constant $C<\infty$ and $k \in \mathbb{N}_{0}$ such that

1991 Mathematics Subject Classification: Primary 46F12.

Key words and phrases: Mellin distributions, Bochner tube theorem. 


$$
|u[\psi]| \leq C \sum_{\substack{|\alpha| \leq k \\ \alpha \in \mathbb{N}_{0}^{n}}} \sup _{\substack{x \in K\\}}\left|\left(\frac{\partial}{\partial x}\right)^{\alpha} \psi(x)\right| \quad \text { for } \psi \in C^{k}\left(\mathbb{R}^{n}\right) .
$$

The proof of this theorem, based on the Whitney extension theorem, is given in [1].

Now following [3] we recall the spaces of Mellin distributions. Denote by $\mu: \mathbb{R}^{n} \rightarrow \mathbb{R}_{+}^{n}$ the diffeomorphism

$$
\mu(y)=e^{-y}:=\left(e^{-y_{1}}, \ldots, e^{-y_{n}}\right) .
$$

We define the space of Mellin distributions on $\mathbb{R}_{+}^{n}$ for every $\alpha \in \mathbb{R}^{n}$ as the dual of the space

$$
\mathfrak{M}_{\alpha}=\mathfrak{M}_{\alpha}\left(\mathbb{R}_{+}^{n}\right)=\left\{\sigma \in C^{\infty}\left(\mathbb{R}_{+}^{n}\right):\left(x^{\alpha+1} \sigma\right) \circ \mu \in S\left(\mathbb{R}^{n}\right)\right\},
$$

with the natural topology in $\mathfrak{M}_{\alpha}$ induced from $S\left(\mathbb{R}^{n}\right)$.

Note that $u \in \mathfrak{M}_{\alpha}^{\prime}\left(\mathbb{R}_{+}^{n}\right)$ if and only if $e^{\alpha y}(u \circ \mu) \in S^{\prime}\left(\mathbb{R}^{n}\right)$. The $M_{\alpha}$ Mellin transform of $u \in \mathfrak{M}_{\alpha}^{\prime}$ is defined by means of the inverse Fourier transform $F^{-1}$ :

$$
M_{\alpha} u=(2 \pi)^{n / 2} F^{-1}\left(e^{\alpha y}(u \circ \mu)\right) .
$$

Here we assume $F \sigma(\xi)=(2 \pi)^{-n / 2} \int_{\mathbb{R}^{n}} e^{-i x \xi} \sigma(x) d x$ for $\xi \in \mathbb{R}^{n}$ and $\sigma \in$ $S\left(\mathbb{R}^{n}\right)$.

For $a \in \mathbb{R}^{n}$ we introduce the space

$$
M_{a}=M_{a}(I)=\left\{\varphi \in C^{\infty}(I): \sup _{x \in I}\left|x^{a+\alpha+\mathbf{1}}(\partial / \partial x)^{\alpha} \varphi(x)\right|<\infty, \alpha \in \mathbb{N}_{0}^{n}\right\}
$$

equipped with the topology defined by the sequence of seminorms

$$
\varrho_{a \alpha}(\varphi)=\sup _{x \in I}\left|x^{a+\alpha+\mathbf{1}}(\partial / \partial x)^{\alpha} \varphi(x)\right|, \quad \alpha \in \mathbb{N}_{0}^{n} .
$$

Note that the space $M_{a}$ is complete (see [3]) but the set $C_{(0)}^{\infty}(I)$ (of restrictions to $I$ of functions in $\left.C_{0}^{\infty}\right)$ is not dense in $M_{a}(I)$.

Let $\omega \in(\mathbb{R} \cup\{\infty\})^{n}$. We define the function space $M_{(\omega)}(I)$ as the inductive limit

$$
M_{(\omega)}(I)=\lim _{a<\omega} M_{a}(I) .
$$

Now the set $C_{(0)}^{\infty}(I)$ is dense in $M_{(\omega)}(I)$ and the dual space $M_{(\omega)}^{\prime}=$ $M_{(\omega)}^{\prime}(I)$ is a subspace of $D_{I}^{\prime}\left(\mathbb{R}_{+}^{n}\right)$. Therefore the elements of $M_{(\omega)}^{\prime}$ are called Mellin distributions on $I$. Note that for $a<b<\omega$ and $\omega \in(\mathbb{R} \cup\{\infty\})^{n}$,

$$
\begin{gathered}
M_{(a)}(I) \subset M_{a}(I) \subset M_{b}(I) \subset M_{(\omega)}(I), \\
M_{(\omega)}(I)=\lim _{a<\omega} M_{(a)}(I), \\
M_{(\omega)}^{\prime}(I)=\bigcap_{a<\omega} M_{a}^{\prime}(I)=\bigcap_{a<\omega} M_{(a)}^{\prime}(I) .
\end{gathered}
$$


The totality of Mellin distributions is denoted by

$$
M^{\prime}(I)=\bigcup_{\omega \in(\mathbb{R} \cup\{\infty\})^{n}} M_{(\omega)}^{\prime}(I)=\bigcup_{\omega \in \mathbb{R}^{n}} M_{(\omega)}^{\prime}(I) .
$$

$M^{\prime}(I)$ coincides with the space of restrictions to $\mathbb{R}_{+}^{n}$ of distributions on $\mathbb{R}^{n}$ with support in $\bar{I}$.

Let $u \in M_{(\omega)}^{\prime}(I)$ for some $\omega \in(\mathbb{R} \cup\{\infty\})^{n}$. We define the Mellin transform of $u$ by

$$
M u(z)=u\left[x^{-z-1}\right] \quad \text { for } \operatorname{Re} z<\omega .
$$

This definition differs from the classical one by the change of variable $z \mapsto-z$.

The following theorem gives a relation between the Mellin transformations $M$ and $M_{\alpha}$ defined by (2) and (1) respectively.

TheOREM 2. Let $u \in M_{(\omega)}^{\prime}(I)$. Then $M u$ is holomorphic for $\operatorname{Re} z<\omega$ and $u \in \mathfrak{M}_{\alpha}^{\prime}\left(\mathbb{R}_{+}^{n}\right)$ for every $\alpha<\omega$. The tempered distribution $M_{\alpha} u$ is a function:

$$
\left(M_{\alpha} u\right)(\beta)=M u(\alpha+i \beta)=(u \circ \mu)\left[e^{(\alpha+i \beta) y}\right] \quad \text { for } \beta \in \mathbb{R}^{n} .
$$

Moreover, $M_{\alpha}: M_{(\omega)}^{\prime} \rightarrow S^{\prime}$ is continuous for $\alpha<\omega$.

Theorem 3 (Paley-Wiener type theorem). In order that a function $f(z)=f\left(z_{1}, \ldots, z_{n}\right)$ be the Mellin transform of a unique Mellin distribution $u \in M_{(\omega)}^{\prime}((0, t])$ it is necessary and sufficient that $f$ be holomorphic in $\left\{z \in \mathbb{C}^{n}: \operatorname{Re} z<\omega\right\}$ and that for every $b<\omega$ and every $\varrho \in \mathbb{R}_{+}$there exist $s=s(b) \in \mathbb{N}_{0}$ and $C=C(b, \varrho)<\infty$ such that

$$
|f(\alpha+i \beta)| \leq C\langle\beta\rangle^{s}\left(e^{\varrho} t\right)^{-\alpha} \quad \text { for } \alpha \leq b .
$$

3. The main theorem. Let $t^{-}=\left(t_{1}^{-}, \ldots, t_{n}^{-}\right), t^{+}=\left(t_{1}^{+}, \ldots, t_{n}^{+}\right), 0<$ $t^{-}<t^{+}$, write $I=\left(0, t^{+}\right]$and consider the polyinterval

$$
\left[t^{-}, t^{+}\right]=\left\{x \in \mathbb{R}^{n}: t^{-} \leq x \leq t^{+}\right\} .
$$

THEOREM 4. Let $f$ be a function holomorphic on $\left\{z \in \mathbb{C}^{n}: \operatorname{Re} z<\right.$ $0\} \cup\left\{z \in \mathbb{C}^{n}: \operatorname{Re} z>0\right\}$ and such that for every $b \in \mathbb{R}^{n}$ with $b<0$ and $\varrho \in \mathbb{R}_{+}$,

$$
\begin{array}{ll}
|f(\alpha+i \beta)| \leq C\langle\beta\rangle^{s}\left(e^{\varrho} t^{+}\right)^{-\alpha} & \text { for } \alpha<b, \\
|f(\alpha+i \beta)| \leq C\langle\beta\rangle^{s}\left(e^{-\varrho} t^{-}\right)^{-\alpha} & \text { for } \alpha>-b,
\end{array}
$$

with some $s=s(b) \in \mathbb{N}_{0}$ and $C=C(b, \varrho)<\infty$. Moreover, assume that the following limits exist in $S^{\prime}\left(\mathbb{R}^{n}\right)$ and are equal:

$$
\lim _{\alpha \rightarrow 0_{-}} f(\alpha+i \cdot)=\lim _{\alpha \rightarrow 0_{+}} f(\alpha+i \cdot) .
$$


Then there exists a unique $u \in D_{\left[t^{-}, t^{+}\right]}^{\prime}$ such that $M u=f$. Furthermore, $f$ is an entire function on $\mathbb{C}^{n}$ and for every $b \in \mathbb{R}^{n}$ and $\varrho \in \mathbb{R}_{+}$there exist $C=C(b, \varrho)<\infty$ and $s=s(b) \in \mathbb{N}_{0}$ such that for any $\sigma \in\{-,+\}^{n}$,

(8) $|f(\alpha+i \beta)| \leq C\langle\beta\rangle^{s}\left(e^{\sigma_{1} \varrho} t_{1}^{\sigma_{1}}\right)^{-\alpha_{1}} \ldots\left(e^{\sigma_{n} \varrho} t_{n}^{\sigma_{n}}\right)^{-\alpha_{n}}$

$$
\text { for } \sigma_{j} \alpha_{j} \leq \sigma_{j} b_{j}, j=1, \ldots, n \text {. }
$$

Proof. By assumption (5), which is the sufficient condition in Theorem 3, there exists a unique distribution $u \in M_{(0)}^{\prime}\left(\left(0, t^{+}\right]\right)$such that $M u=f$. Thus supp $u \subset\left(0, t^{+}\right]$and $u \in \mathfrak{M}_{\alpha}^{\prime}\left(\left(0, t^{+}\right]\right)$for $\alpha<0$. Denote by $w$ the tempered distribution defined by $(7)$. Hence

$$
\lim _{\alpha \rightarrow 0_{-}} \int_{\mathbb{R}^{n}} f(\alpha+i \beta) \psi(\beta) d \beta=w[\psi] \quad \text { for } \psi \in S\left(\mathbb{R}^{n}\right)
$$

and by (3) and (1) we get

$$
\begin{aligned}
w[\psi] & =\lim _{\alpha \rightarrow 0_{-}} \int_{\mathbb{R}^{n}}(M u)(\alpha+i \beta) \psi(\beta) d \beta \\
& =(2 \pi)^{n / 2} \lim _{\alpha \rightarrow 0_{-}} \int_{\mathbb{R}^{n}} F^{-1}\left(e^{\alpha y}(u \circ \mu)\right)(\beta) \psi(\beta) d \beta \\
& =(2 \pi)^{n / 2} \lim _{\alpha \rightarrow 0_{-}} F^{-1}\left(e^{\alpha y}(u \circ \mu)\right)[\psi] \\
& =(2 \pi)^{n / 2} \lim _{\alpha \rightarrow 0_{-}}\left(e^{\alpha y}(u \circ \mu)\right)\left[F^{-1} \psi\right] \\
& =(2 \pi)^{n / 2} \lim _{\alpha \rightarrow 0_{-}}(u \circ \mu)\left[e^{\alpha y} F^{-1} \psi\right] .
\end{aligned}
$$

For $\psi=F \varphi$ with $\varphi \in D\left(\mathbb{R}^{n}\right)$ the last formula yields

$$
F w[\varphi]=(2 \pi)^{n / 2}(u \circ \mu)[\varphi] \quad \text { for } \varphi \in D\left(\mathbb{R}^{n}\right) .
$$

Now observe that by assumption (6),

$$
|f(-\alpha-i \beta)|<C\langle\beta\rangle^{s}\left(e^{\varrho} \frac{1}{t^{-}}\right)^{-\alpha} \quad \text { for } \alpha<b,
$$

where $1 / x:=\left(1 / x_{1}, \ldots, 1 / x_{n}\right)$ for $x \in \mathbb{R}_{+}^{n}$. As before, by Theorem 3 , there exists a unique distribution $\widetilde{u} \in M_{(0)}^{\prime}\left(\left(0,1 / t^{-}\right]\right)$such that

$$
f(-\alpha-i \beta)=M \widetilde{u}(\alpha+i \beta) .
$$

Note that $\widetilde{u} \in \mathfrak{M}_{\alpha}^{\prime}\left(\left(0,1 / t^{-}\right]\right)$for $\alpha<0$ and $f(-\alpha-i \beta)=M_{\alpha} \widetilde{u}(\beta)=$ $(2 \pi)^{n / 2} F^{-1}\left(e^{\alpha y}(\widetilde{u} \circ \mu)\right)$.

Since by $(7), w=\lim _{\alpha \rightarrow 0_{+}} f(\alpha+i \cdot)$ we have $\lim _{\alpha \rightarrow 0_{-}} f(-\alpha-i \cdot)=w^{\vee}$ where ${ }^{\vee}$ denotes the reflection $\beta \rightarrow-\beta$. Take $\varphi \in D\left(\mathbb{R}^{n}\right)$ and let $\psi=F \varphi$. 
Then $\psi \in S\left(\mathbb{R}^{n}\right)$ and

$$
\begin{aligned}
w^{\vee}[\psi] & =\lim _{\alpha \rightarrow 0-} \int_{\mathbb{R}^{n}} f(-\alpha-i \beta) \psi(\beta) d \beta \\
& =(2 \pi)^{n / 2} \lim _{\alpha \rightarrow 0_{-}} \int_{\mathbb{R}^{n}} F^{-1}\left(e^{\alpha y}(\widetilde{u} \circ \mu)\right) \psi(\beta) d \beta=(2 \pi)^{n / 2}(\widetilde{u} \circ \mu)[\varphi] .
\end{aligned}
$$

Hence

$$
F w^{\vee}[\varphi]=(2 \pi)^{n / 2}(\widetilde{u} \circ \mu)[\varphi] \quad \text { for } \varphi \in D\left(\mathbb{R}^{n}\right) .
$$

This together with (9) yields $(u \circ \mu)^{\vee}=\widetilde{u} \circ \mu$. Let $\lambda$ be the mapping $\mathbb{R}_{+}^{n} \ni x \mapsto$ $1 / x$. Since $(u \circ \mu)^{\vee}=(u \circ \lambda) \circ \mu$ we get $u \circ \lambda=\widetilde{u}$. Hence $u \circ \lambda \in M_{(0)}^{\prime}\left(\left(0,1 / t^{-}\right]\right)$ and by definition of $\lambda$ we have $\operatorname{supp} u \subset\left\{x: x \geq t^{-}\right\}$, which together with $u \in M_{(0)}^{\prime}\left(\left(0, t^{+}\right]\right)$gives the desired assertion $u \in D_{\left[t^{-}, t^{+}\right]}^{\prime}$. By Theorem $1, u \in M_{a}^{\prime}$ for every $a \in \mathbb{R}^{n}$ (i.e. $\left.u \in M_{(\infty)}^{\prime}\right)$ and hence by Theorem 3 (the necessary condition this time) $f=M u$ is entire on $\mathbb{C}^{n}$ and the estimate (5) holds for $\alpha \leq b$ for every $b \in \mathbb{R}^{n}$. Since $u \circ \lambda \in D_{\left[1 / t^{+}, 1 / t^{-}\right]}^{\prime}$ and $M(u \circ \lambda)(z)=M u(-z)$ we get as before, for all $b \in \mathbb{R}^{n}$ and $\varrho \in \mathbb{R}_{+}$,

$$
|M u(\alpha+i \beta)| \leq C\langle\beta\rangle^{s}\left(e^{-\varrho} t^{-}\right)^{-\alpha} \quad \text { for } \alpha \geq b
$$

with $s=s(b)$ and $C=C(b, \varrho)$. Thus we have proved $(8)$ for $\sigma=(+, \ldots,+)$ and $\sigma=(-, \ldots,-)$. To get the proof for $\sigma^{j}=\left(\sigma_{1}^{j}, \ldots, \sigma_{n}^{j}\right)$ with $\sigma_{i}^{j}=+$ if $i \neq j$, and $\sigma_{j}^{j}=-1(j=1, \ldots, n)$, take the mapping

$$
\lambda_{j}: \mathbb{R}_{+}^{n} \ni x \mapsto\left(x_{1}, \ldots, x_{j-1}, 1 / x_{j}, x_{j+1}, \ldots, x_{n}\right) .
$$

Then

$$
\begin{gathered}
M\left(u \circ \lambda_{j}\right)(z)=M u\left(z_{1}, \ldots, z_{j-1},-z_{j}, z_{j+1}, \ldots, z_{n}\right) \\
=f\left(z_{1}, \ldots, z_{j-1},-z_{j}, z_{j+1}, \ldots, z_{n}\right), \\
\operatorname{supp}\left(u \circ \lambda_{j}\right) \subset\left\{x: t_{i}^{-} \leq x_{i} \leq t_{i}^{+} \text {for } i \neq j, 1 / t_{j}^{+} \leq x_{j} \leq 1 / t_{j}^{-}\right\} .
\end{gathered}
$$

Fix arbitrarily $b \in \mathbb{R}^{n}, \varrho \in \mathbb{R}_{+}$and $j$ with $1 \leq j \leq n$. Take $\widetilde{b}=\left(b_{1}, \ldots, b_{j-1}\right.$, $\left.-b_{j}, b_{j+1}, \ldots, b_{n}\right)$. By Theorem 3 ,

$$
\left|M\left(u \circ \lambda_{j}\right)(\alpha+i \beta)\right| \leq C\langle\beta\rangle^{s}\left(e^{\varrho} t_{1}^{+}\right)^{-\alpha_{1}} \ldots\left(e^{\varrho} \frac{1}{t_{j}^{-}}\right)^{-\alpha_{j}} \ldots\left(e^{\varrho} t_{n}^{+}\right)^{-\alpha_{n}}
$$

for $\alpha \leq \widetilde{b}$ and hence

$$
|f(\alpha+i \beta)| \leq C\langle\beta\rangle^{s}\left(e^{\varrho} t_{1}^{+}\right)^{-\alpha_{1}} \ldots\left(e^{-\varrho} t_{j}^{-}\right)^{-\alpha_{j}} \ldots\left(e^{\varrho} t_{n}^{+}\right)^{-\alpha_{n}}
$$

for $\alpha_{i} \leq b_{i}$ if $i \neq j,-\alpha_{j} \leq \widetilde{b}_{j}=-b_{j}$, i.e. (8) holds for $\sigma^{j}(j=1, \ldots, n)$.

The remaining cases of $\sigma$ are left to the reader.

Remark 1. Note that in contrast to the classical Paley-Wiener theorem the sufficiency part of Theorem 4 does not require that the function 
$f$ be entire. Instead we assume holomorphy in two wedges with a common edge and identity of the corresponding boundary values. By the necessity result this gives holomorphy in $\mathbb{C}^{n}$ as well as estimates in the "missing" wedges, which can be regarded as a variant of the Bochner tube theorem.

Remark 2. By applying the techniques of the theory of Fourier hyperfunctions and analytic functionals one can prove a variant of Theorem 4 with $\langle\beta\rangle^{s}$ in the estimates (5), (6) and (8) replaced by $e^{\theta|\beta|}$ for some $\theta>0$. Then the identity (7) should be understood as the equivalence of pertinent boundary values in the sense of Fourier hyperfunctions.

\section{References}

[1] L. Hö r m a n d er, The Analysis of Linear Partial Differential Operators I, Springer, 1985.

[2] W. Rudin, Lectures on the Edge of the Wedge Theorem, Amer. Math. Soc., Providence, 1971.

[3] Z. S z m y d t and B. Z i e m i a n, The Mellin Transformation and Fuchsian Type Partial Differential Equations, Math. Appl. 56, Kluwer, 1992.

INSTITUTE OF MATHEMATICS

POLISH ACADEMY OF SCIENCES

ŚNIADECKICH 8

00-950 WARSZAWA, POLAND

Reçu par la Rédaction le 2.3.1994 